\title{
Modelling of High Dynamic Range Logarithmic CMOS Image Sensors
}

\author{
Stephen O. Otim, Dileepan Joseph, Bhaskar Choubey, Steve Collins. \\ Department of Engineering Science, \\ University of Oxford, OX13PJ, Oxford, United Kingdom \\ Phone: +44(0)1865273805, Fax: +44(0)1865273905 Email: otimso@ robots.ox.ac.uk.
}

\begin{abstract}
The quality of the output images from high dynamic range logarithmic sensors is limited by fixed pattern noise (FPN) which is caused by device mismatches within pixels in an array. It leads to inferior image quality in comparison to images from other sensors of similar resolution. Previous design and post-chip attempts to correct this type of noise, have been either impractical or resulted in other complexities. However, FPN correction can be attempted using an accurate model approach for the response of this type of pixel. A three parameter model, previously suggested for logarithmic pixels, has been tried for this purpose. In this paper a simple parameter extraction procedure is proposed using this model to calibrate and correct FPN. The result is a model that works well over six decades of illumination but fails at high photocurrents. It is shown that this is caused by a breakdown in an assumption used to create the three parameter model. Consequently, a new four parameter model is developed that fits the data over six decades, and is usable in FPN correction for many wide current range applications that require complete and accurate characterisation.
\end{abstract}

Keywords - EKV Model, Function Minimisation, CMOS Logarithmic Sensors, Fixed Pattern Noise (FPN) Correction, CMOS Sensor Calibration.

\section{INTRODUCTION}

Real world scenes have dynamic intra-scene ranges that might extend about five orders of magnitude [1], from 1 lux in shadows to $10^{5}$ lux in bright sunlight. Unfortunately, Charged coupled devices (CCD's) and Active pixel sensors (APS) [2], which currently dominate the image sensor market have a dynamic range of less than three orders of magnitude. Consequently, when imaging natural and industrial scenes the response of these sensors saturates in parts of the scene. To overcome these problems several on-chip techniques have been proposed that can extend the dynamic range of the sensor. Most of these techniques including multi-sampling, threshold detection, integration time control [3], [4], [5] are based upon pixels with a linear response and any dynamic range improvement can only be achieved by a significant increase in the number of bits per pixel. This leads to a further increase in clock signals, circuitry and computational needs in addition to slowing sensor operation in fast frame rate applications.

One way of avoiding these complications is to use another approach to capturing high dynamic range scenes by changing the pixel circuit so that it has a logarithmic response. Such a sensor can be designed using a MOS transistor operating in the subthreshold region. These pixels compress the dynamic range of the input signal whilst preserving the contrast information that is important to both users and image recognition systems. The problem with logarithmic pixels is that the quality of the resulting output image is severely degraded by fixed pattern noise (FPN) caused by mismatches between the components of individual pixels in each sensor [6], [7]. FPN appears as pixels having different responses when exposed to the same illumination hence these images are inferior to those presented by linear sensors of comparable resolutions. In addition, the continuous nature of operation of logarithmic sensors, makes it impossible to employ FPN reduction methods like double sampling used in CCD and APS linear sensors [8]. To alleviate this problem a three parameter model for a logarithmic pixel has been developed by Joseph and Collins [9] that can be used to correct the response of each pixel and hence remove fixed pattern noise. They devised an off-chip calibration technique based on the three parameter model which uses 24 images to completely calibrate a logarithmic sensor.

This paper further develops this model by introducing a simple three parameter extraction procedure, which requires only three readings for complete FPN correction. The model has been further studied for very bright scenes and it has been observed that the pixel response deviates from the model responses for such scenes. The reasons for such a discrepancy have also been studied in this paper and a new four parameter model has been proposed to account for this deviation. The rest of the paper is arranged as follows.

The circuit for a typical logarithmic pixel and the three parameter model for its response is described in detail in section II. This is followed in section III by a description of a simple procedure to extract the three parameters that are required to represent the response of individual pixels. Results show that this procedure works well over at least four decades of input photocurrent. However, the difference between the simulated pixel response and the modelled response increases rapidly at high photo-currents. This deviation between the predicted and actual response of the pixel is then explained by introducing a four parameter model which is derived from the EKV transistor model in section IV. Finally, a conclusion of 
the work contained in this paper is made in section V.

\section{BACKGROUND}

A typical logarithmic pixel circuit is shown in figure 1. In this pixel, light falling on the photodiode causes a current to flow through transistor $T 1$. When this device is operating in weak inversion it creates a logarithmic relationship between the transistor current $I_{d}$ and the voltage $V_{g}^{T 2}$ on the gate of transistor $T 2$ as shown below.

$$
I_{d}=I_{d s} \exp \left(\frac{V_{g s}^{T 2}-V_{o n}^{T 1}}{n K T / q}\right)
$$

where $I_{d s}$ is the drain current when the transistor saturates, $n$ is the free electron concentration, $K, T$ and $q$ are the usual constants in transistor theory. Transistors $T 2, T 3$ and $T 4$ then form an nMOS source follower circuit that can be used to selectively connect the pixel to a column output bus. Finally, transistors $T 5, T 6$ and $T 7$ form a pMOS source follower that connect a particular column output to the sensor output bus for digitisation.

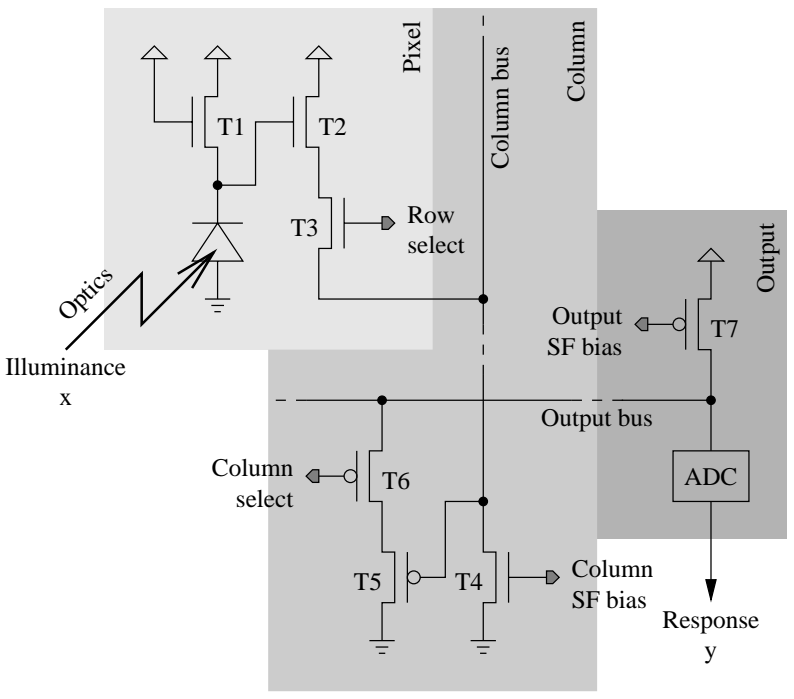

Fig. 1. Pixel Structure: From an illuminance $x$ to a digital response $y$ in one pixel of a logarithmic CMOS image sensor. Transistors $T 2$ with $T 4$ and $T 5$ with $T 7$ form an nMOS and pMOS source follower (SF) respectively, when $T 3$ and $T 6$ are turned on.

Assuming that the two source-follower circuits are ideal then the response of a pixel $y$ to a photo-current $x$ can be represented by the equation 1

$$
y=a+b \ln (x+c)
$$

where $a$ represents an additive offset voltage, $b$ is the gain of the pixel and $c$ represents the effect of a leakage current within the pixel. Joseph and Collins [9], [10] have shown that this equation can be used to represent the response of the pixels in a logarithmic sensor and that variations in all parameters contribute to fixed pattern noise. However, to prove the validity of the model and determine the limit of its accuracy they used a parameter extraction procedure based upon iterative parameter estimation using twenty four different images. Predictably this method was inconvenient to users as it required several images in different illumination conditions to calibrate the sensor in addition to the heavy computational demand on the camera system. This is particularly cumbersome in fast rate applications like in defense and the industrial sectors where input data is used for further decision making.

\section{SIMPLE PARAMETER EXTRACTION}

\section{A. Theory}

To overcome the impractical nature of the parameter extraction procedure used by Joseph and Collins, a simpler procedure is required that is both computationally less demanding and requires less data. The three parameter model in equation 1 has three coefficients, hence a minimum of three data points is required to solve for unknowns $a, b, c$. In creating a simple parameter extraction, the calibration data points should be chosen carefully. In particular, the non-linear transfer function of equation 1 can be simplified at high illumination when the photocurrent becomes much higher than leakage current and hence $c$ can be safely neglected. This reduces the model equation to

$$
y=a+b \ln (x)
$$

Using this approximation pixel responses, $y_{1}$ and $y_{2}$, at two different bright illuminances $x_{1}$ and $x_{2}$, can be used to calculate the gain and offset parameter of each pixel using the equations

$$
\begin{aligned}
b & =\frac{y_{1}-y_{2}}{\ln \left(x_{1} / x_{2}\right)} \\
a & =y_{1}-b \ln \left(x_{1}\right)
\end{aligned}
$$

With these two parameters, the third coefficient, $c$, can be determined from the dark response of the pixel, $y_{d}$ using;

$$
c=\exp \left(\frac{y_{d}-a}{b}\right)
$$

Thus, using these three data points i.e. the dark reading and two bright readings, the coefficients that form the response of each pixel can be calculated leading to the complete calibration and characterisation of a logarithmic sensor.

\section{B. Simulation}

The accuracy of the three parameter model, when equations 2, 3 and 4 were used to extract these parameters, has 


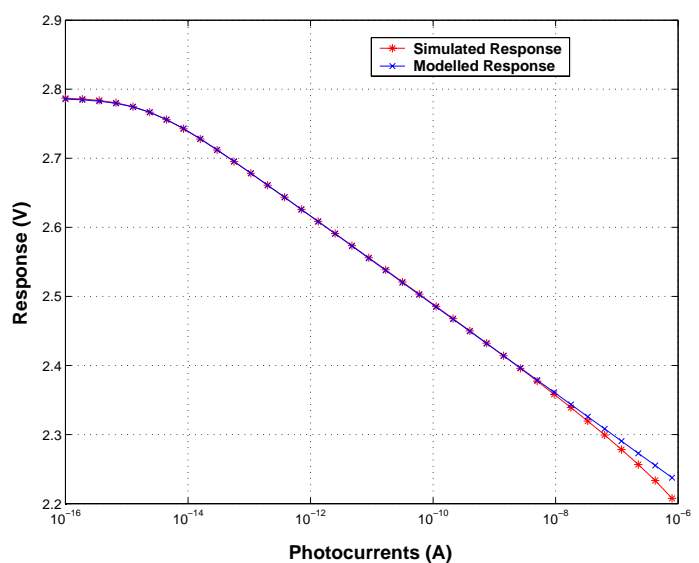

Fig. 2. A comparison of the responses of a logarithmic pixel obtained from circuit simulation software and the model response when the model parameters were obtained using the simple parameter extraction procedure on the three parameter model.

been investigated using data obtained from a circuit simulator. A logarithmic pixel designed in a $0.35 \mu \mathrm{m}$ AMS CMOS process with device geometries chosen to represent a $10 \mu \mathrm{m}$ by $10 \mu m$ pixel with a fill factor of $58 \%$ was used to obtain this simulation data. Results from the simulator showed that the output voltage from the pixel changed by $65 \mathrm{mV}$ when the photocurrent increases by a decade of magnitude.

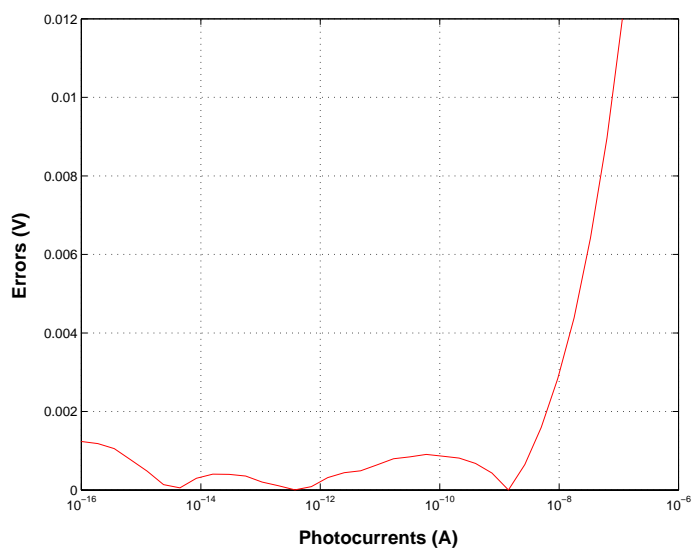

Fig. 3. Errors obtained as the difference between the simulated response of a logarithmic pixel and the modelled response using the simple parameter extraction procedure on the three parameter model.

The predicted pixel response after simple parameter extraction and the data from the circuit simulator are both shown in figure 2. Clearly, the model fits the data very well at photocurrents less than $1 n A$. The error plots in figure 3 show that in this region the error between the predicted and the actual response is about one millivolt. This means the data has been fitted to the nearest millivolt which is an excellent fit considering that the data used was also accurate to the nearest millivolt. However, both figures clearly show that for photocurrents more than
$1 n A$, the predicted and the actually responses diverge rapidly. In this region the model would not be able to correct for fixed pattern noise effectively.

\section{Discussion}

There are two possible reasons why the model might fail to predict the pixel response at high current levels. The simple parameter extraction routine could be extracting incorrect parameter values that cause large errors when the model is extrapolated to high current values. Alternatively, the assumption upon which the model is based that the load transistor is operating in weak inversion could be breaking down as high photocurrents force the device to operate with a source-gate voltage close to its threshold voltage.

To test the accuracy of the simple parameter extraction routine, its results need to be compared to a more sophisticated and accurate procedure. Since the three parameter model is a non-linear model, the parameters from the model were obtained using a function minimisation scheme. In this search technique, the user provides a range of possible values for the non-linear parameter $c$, that is likely to contain the actual value of $c$. For all the values of $c$ in this range, a linear optimisation technique is used to determine the values of both $a$ and $b$. The sum square error (SSE) between the results of the model with the extracted parameters and the simulated responses is then calculated for each value of $c$. The most accurate value of the model coefficients gives the minimum error between the estimated response and the modelled response. Ideally the best value of $c$ will give a zero sum square error. In practice, the parameter values taken are those that correspond to a minimum SSE [9], for the user provided range.

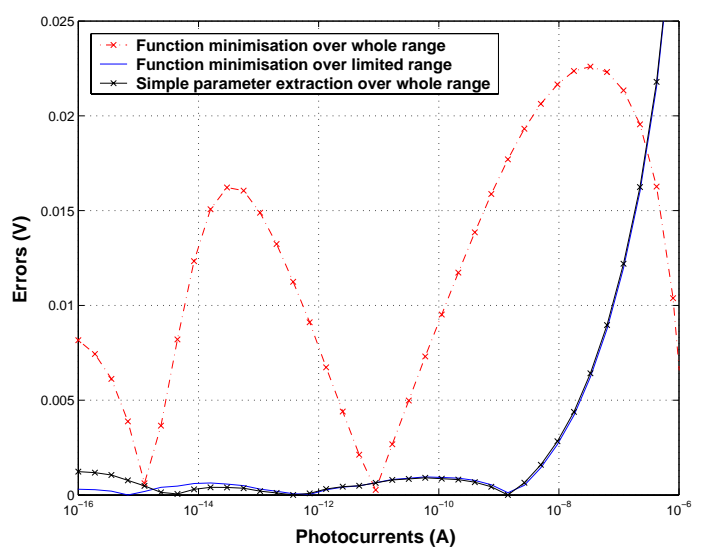

Fig. 4. The comparative errors in the three parameter model when two different parameter extraction procedures are used over varying photocurrent ranges.

The parameters from the model were therefore obtained using this regressive function minimisation method with data 
over the whole current range and then over a limited lower photocurrent range ${ }^{1}$. A comparison of the accuracy of the three parameter model when its parameters have been extracted using these two different techniques is shown in figure 4 . The more sophisticated function minimisation technique, restricted to less than $1 n A$, gives comparable results to the simple parameter extraction method over the same region. However, the same function minimisation gives very large errors when higher currents are considered over the whole range. This seems to be caused by the extraction algorithm trying to find a versatile solution for both the low and high current ranges. When combined with the rapid increase in model error at high photo-currents observed for both parameter extraction techniques this result leads to the conclusion that the model is inaccurate at high photocurrents.

\section{A FOUR PARAMETER MODEL}

The three parameter model developed previously, begins to fail in the region of high photocurrents. This could be because at high photocurrents the pixel load transistor, $T 1$ in figure 1 , is being forced to operate in moderate rather than weak inversion. If this is the case then the assumption of a logarithmic relationship between photo-current and source-gate voltage used to derive equation 1 becomes invalid.

To test this possibility a model for the response of the load transistor $T 1$ is required that is valid when this device is operating in both weak and moderate inversion. A model that is very suitable for this purpose is the Enz-Krummenacher-Vittoz (EKV) MOS transistor model [11]. The advantage of the EKV model is that it is based upon a single function that represents all operating regions of the transistor from weak to strong inversion. In this model the drain-source current, $I_{D S}$, in a transistor of width $W$ and length $L$ with an effective capacitance per unit area $C_{o x}^{\prime}$ can be written in the form [12]

$$
I_{D S}=\frac{W}{L} \mu C_{o x}^{\prime} 2 n \phi_{t}^{2}\left[\ln \left(1+\exp ^{\left(V_{G S}-V_{t}\right) / 2 n \phi_{t}}\right)\right]^{2}
$$

where $\mu$ is the effective surface mobility, $V_{t}$ is the transistor threshold voltage, $\phi_{t}$ is the thermal voltage, and $n$ is the subthreshold slope parameter.

Within the pixel, the drain-source current of the load transistor T1 is the sum of the photocurrent $I_{p}$ and the leakage current $I_{s}$. Now rearranging equation 5 to obtain an expression for the gate voltage of transistor T2, and setting $I_{D S}=I_{p}+I_{s}$, gives,

$$
V_{G S}=V_{t}+2 n \phi_{t} \ln \left[\exp \sqrt{\frac{L}{W \mu C_{o x}^{\prime} 2 n \phi_{t}^{2}}\left(I_{p}+I_{s}\right)}-1\right]
$$

Then assuming the source follower circuits are ideal this can be written in the form,

$$
y=a+b \ln \left(\exp ^{\sqrt{c+d x}}-1\right)
$$

1 The extraction technique was tried for currents lower than 1 Amps
Again $a$ represents an offset in the output voltage, $b$ represents the pixel gain and $c$ the effects of the leakage current in the pixel. In addition, there is now a fourth parameter $d$, which scales the input photocurrent. Equation 6 is a general equation that can be used to model the response $y$ of a pixel with a photocurrent $x$ flowing through its photodiode. At low photocurrents, when the load transistor is operating in weak inversion, this expression should reduce to the three parameter model. To show how this occurs, consider parameters $d$ and $c$ and the photocurrent $x$. The parameters $d$ and $c$ are given by the equation

$$
\begin{aligned}
& d=\frac{L}{W \mu C_{o x}^{\prime} 2 n \phi_{t}^{2}} \\
& c=d I_{s}
\end{aligned}
$$

Comparing the equation for $d$ to the simple model of a transistor operating in saturation, with a source-gate voltage greater than the threshold voltage,

$$
I_{D S}=\frac{W}{2 L} \mu C_{O X}^{\prime}\left(V_{g s}-V_{t}\right)^{2}
$$

suggests that this parameter is the inverse of the scaled current that will flow through the device when it is biased just above its threshold voltage. The current flowing through a device biased near its threshold voltage is much larger than the leakage current through the pixel, the parameter $c$ is therefore much less than unity. Furthermore, for the load transistor to be operating in subthreshold the photocurrent will also be less than this current and hence $d x<1$. Under these conditions

$$
\exp ^{(\sqrt{c+d x})} \approx 1+(\sqrt{c+d x})
$$

and hence

$$
\begin{aligned}
& y=\left(a+\frac{b}{2} \ln (d)\right)+\frac{b}{2} \ln \left(\frac{c}{d}+x\right) \\
& y=\dot{a}+\dot{b} \ln (\dot{c}+x)
\end{aligned}
$$

Equation 7 is the same form as the three parameter model, equation 1, that has been seen to represent the response of the logarithmic pixels at low photocurrents. As expected the four parameter model therefore reduces to the simpler three parameter model. In addition, the conditions for this simplification are those that ensure that the load transistor is operating in weak inversion as assumed in developing the three transistor model. The four parameter model is therefore a more general and accurate model for the characterisation of a logarithmic pixel over a wider range of photocurrents.

As with the three parameter model, the four parameter model response was compared with the output of a circuit simulation of a logarithmic pixel. Again the parameters for a non-linear model were obtained using functional minimisation. The results of comparing the response of the logarithmic pixel with the resulting model is shown in figure 5 while the errors between the modelled and simulated responses are shown 


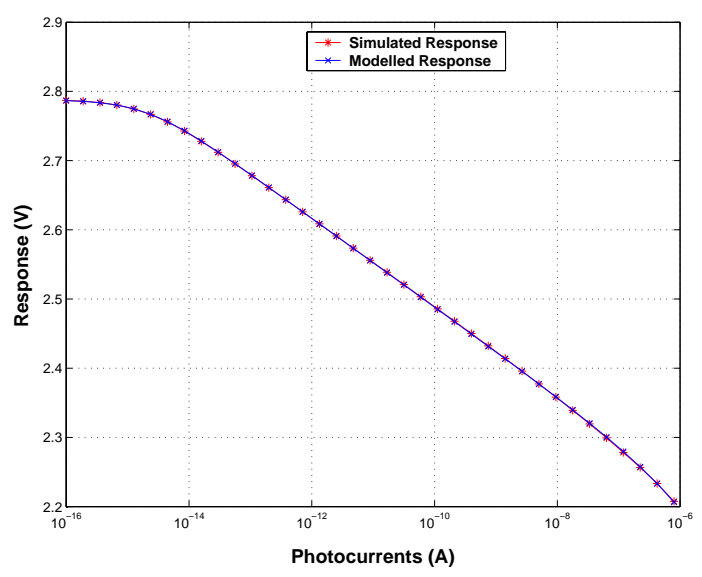

Fig. 5. A comparison between the responses of a logarithmic pixel obtained from circuit simulation software for the four parameter model.

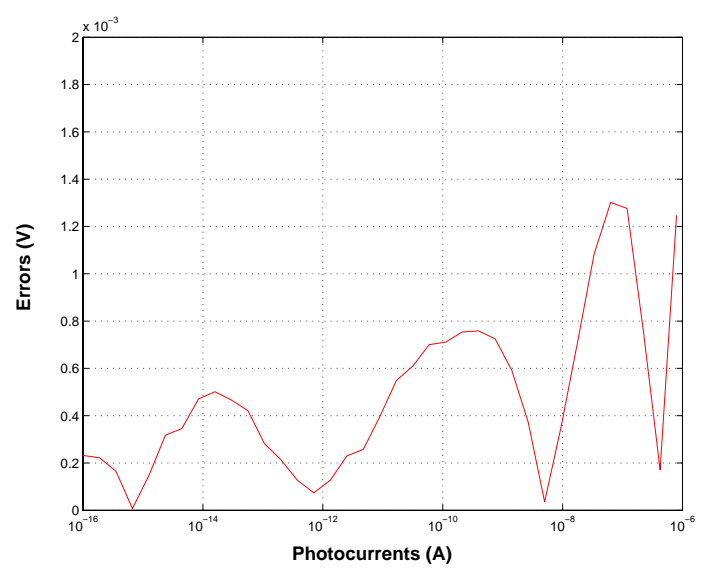

Fig. 6. The Comaprative eErrors between the response of a logarithmic pixel obtained from circuit simulation software for the four parameter model

in figure 6. The results obtained, showed that the four parameter model fits the data in the high current region in which the three parameter model gave rise to large errors. For the whole range of different input currents, the four parameter model fitted the response of the logarithmic pixel to less than one millivolt which corresponds to the least significant bit of the data being fitted.

Using the four parameter model and the values of its various parameters it is now possible to split the response of the logarithmic pixel into three regions, shown in figure 7. At low photo-currents the response of the pixel is largely determined by the bias parameter which arises from leakage currents within the pixel. As the photocurrent increases the pixel enters the region in which its response is proportional to the logarithm of the photocurrent. Finally, at high photocurrents the load transistor is driven into moderate inversion and the logarithmic relationship breaks down. The errors in the three parameter model at high photocurrents therefore arise because of the limited range of validity of the model rather than from any error in the simple parameter extraction routine.

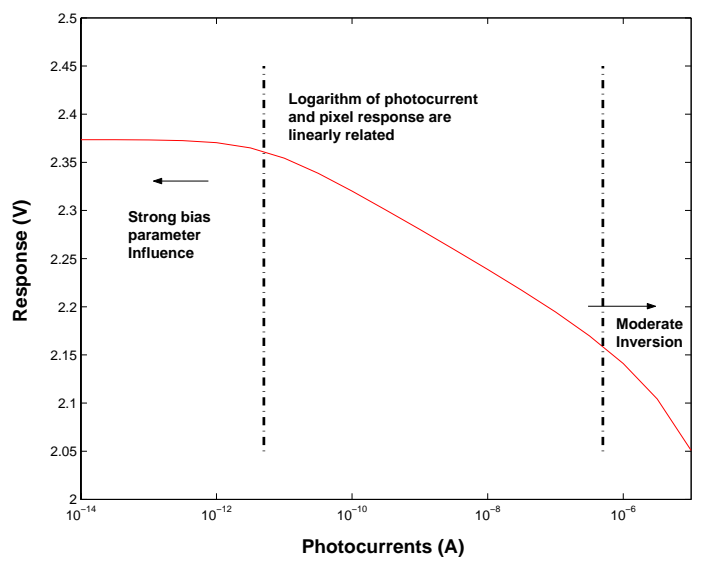

Fig. 7. The different regions of operation of a pixel exposed to a wide range of illuminations.

\section{CONCLUSION}

Based on the three parameter model of the response of a logarithmic pixel previously proposed by Joseph and Collins, a simple procedure to estimate the parameters for this model has been proposed that uses only three data points and three simple equations. As expected with a simple procedure the error between the model and the response of a logarithmic pixel was slightly larger than one least significant bit of the digitised data. The more surprising result was that this procedure highlighted a divergence between the model and the pixel response at photocurrents larger than $10 \mathrm{nA}$ that had not been observed previously. Using a more sophisticated parameter estimation technique, it has been shown that the divergence between the three parameter model and the pixel response at high photocurrents arises from a problem with the three parameter model. A four parameter model has therefore been developed that can represent the load transistor in moderate inversion as well as weak inversion. This four parameter model was then shown to fit the response of a pixel circuit over at least six decades, covering three different modes of operation. This new model may be needed to correct fixed pattern noise for applications that require the pixel to operate over very high dynamic ranges. Alternatively, it may be needed in applications that require precise estimates of the actual photocurrent, such as might be required in colour sensors. However, our experience suggests that noise and interference currently limit the quality of the output from most logarithmic sensors to a level that would limit the benefits of using this model. For these sensors the simpler three parameter model can be used to reduce fixed pattern noise to below the level of these temporal noise processes. Once this 
is achieved it is the temporal noise that will limit the quality of the resulting image.

\section{REFERENCES}

[1] Robert M. Boynton, Human Colour Vision, University of California, San Diego, 1979.

[2] Keith Diefendorff, "CMOS Image Sensors Challenge CCD's," Microprocessor Report, pp. 1-5, 22 June 1998, Micro Design Resources.

[3] A. Bubmann B J Hostika M. Schanz, C. Nitta and R K Wertheimer., "A high dynamic range cmos image sensor for automotive applications.," IEEE Journal on Solid State Circuits, vol. 35, no. 7, pp. 932-938, July 2000.

[4] O. Yadid-Pecht and E. R Fossum., "Image sensor with ultra high linear dynamic range utilising dual output cmos active pixel sensors," IEEE Transactions on Electron Devices, vol. 44, no. 10, pp. 1721-1723, October 1997.

[5] L. Gonzo D. Stopa, A. Simoni and G. Dalla Betta., "Novel cmos image sensor with a 132-db dynamic range.," IEEE Journal on Solid State circuits, vol. 37, no. 12, pp. 1846-1852, December 2002.

[6] Markus Loose, Karlheinz Meier, and Johannes Schemmel, "CMOS image sensor with logarithmic response and self calibrating fixed pattern noise correction," in Proceedings of the SPIE, May 1998, vol. 3410, pp. 117-27, Advanced Focal Plane Arrays and Electronic Cameras II.

[7] Markus Loose, Karlheinz Meier, and Johannes Schemmel, "A SelfCalibrating Single-Chip CMOS Camera with Logarithmic Response," IEEE Journal of Solid-State Circuits, vol. 36, no. 4, pp. 586-96, Apr. 2001.

[8] Sunetra K. Mendis, Sabrina E. Kemeny, Russell C. Gee, Bedabrata Pain, Craig O. Staller, Quiesup Kim, and Eric R. Fossum, "CMOS Active Pixel Image Sensors for Highly Integrated Imaging Systems," IEEE Journal of Solid-State Circuits, vol. 32, no. 2, pp. 187-97, Feb. 1997.

[9] Joseph Dileepan and Steve Collins, "Modelling, calibration and correction of non-linear illumination dependent fixed pattern noise in logarithmic cmos sensors.," Transactions on Instrumentation and Measurement, vol. 51, no. 5, pp. 996-1001, October 2002.

[10] Joseph Dileepan and Steve Collins, "Modelling, calibration and rendition of colour logarithmic cmos image sensors.," Transactions on Instrumentation and Measurement, vol. 52, no. 5, pp. 1581-1587, October 2003.

[11] E Vittoz C. Enz, F.Krummenacher, "An analytical mos transistor model valid in all regions of operation and dedicated to low voltage and low current applications.", Analogue integrated circuits and signal processing, pp. 83-114, July 1995.

[12] Yannis Tsividis, Operation and Modelling of The MOS Transistor, McGraw Hill, second edition, 1999, Pg 178. 